DOE/NV/25946--498

\title{
Real-time X-ray Diffraction Measurements of Shocked Polycrystalline Tin and
}

\author{
Aluminum \\ Dane V. Morgan, ${ }^{\text {a) }}$ Don Macy, Gerald Stevens
}

National Security Technologies, LLC, Los Alamos Operations, 182 East Gate Drive, Los

Alamos, NM 87544

a) Author to whom correspondence should be addressed. Electronic mail: morgandv@nv.doe.gov. 
A new, fast, single-pulse x-ray diffraction (XRD) diagnostic for determining phase transitions in shocked polycrystalline materials has been developed. The diagnostic consists of a 37-stage Marx bank high-voltage pulse generator coupled to a needle-andwasher electron beam diode via coaxial cable, producing line and bremsstrahlung x-ray emission in a 35-ns pulse. The characteristic $\mathrm{K}_{\alpha}$ lines from the selected anodes of silver and molybdenum are used to produce the diffraction patterns, with thin foil filters employed to remove the characteristic $\mathrm{K}_{\beta}$ line emission. The $\mathrm{x}$-ray beam passes through a pinhole collimator and is incident on the sample with an approximately $3-\mathrm{mm}$ by $6-\mathrm{mm}$ spot and $1^{\circ}$ full-width-half-maximum (FWHM) angular divergence in a Bragg-reflecting geometry. For the experiments described in this report, the angle between the incident beam and the sample surface was $8.5^{\circ}$. A Debye-Scherrer diffraction image was produced on a phosphor located $76 \mathrm{~mm}$ from the polycrystalline sample surface. The phosphor image was coupled to a charge-coupled device (CCD) camera through a coherent fiberoptic bundle. Dynamic single-pulse XRD experiments were conducted with thin foil samples of tin, shock loaded with a 1-mm vitreous carbon back window. Detasheet high explosive with a 2-mm-thick aluminum buffer was used to shock the sample. Analysis of the dynamic shock-loaded tin XRD images revealed a phase transformation of the tin beta phase into an amorphous or liquid state. Identical experiments with shock-loaded aluminum indicated compression of the face-centered-cubic (fcc) aluminum lattice with no phase transformation. 


\section{Introduction}

Dynamic x-ray diffraction (XRD) has been widely used to investigate the properties of shocked single-crystal structural dynamics over the past four decades, on time scales of less than 100 ns. However, dynamic studies with shocked polycrystalline materials have been problematic due to insufficient monochromatic x-ray flux and, until recently, dynamic experiments using x-ray diffraction diagnostics have been limited to materials that can be fabricated as single-crystal targets. Dynamic shock studies of polycrystalline materials with dynamic single-pulse XRD diagnostics are a significant advance in this field, allowing polycrystalline melt and solid-solid phase transition experiments to be observed on a very short time scale. Also, the effects of dynamic shocks on material properties including temperature ${ }^{1}$, texture, and grain size distribution caused by dynamic shocks may be investigated using this diagnostic ${ }^{2}$.

Nearly 40 years ago, Johnson et al. ${ }^{3}$ showed that a Debye-Scherrer XRD pattern could be obtained from lithium fluoride $(\mathrm{LiF})$ and sodium chloride powder samples with 2.75- $\AA$ x-rays in less than 100 ns with a Blumlein pulse generator. To obtain these results, the detector was placed within three centimeters of the sample, making dynamic experiments impractical due to difficulties protecting the soft x-ray detector from shrapnel. Later, Johnson et al. used pulsed x-ray diffraction diagnostics to perform dynamic experiments with single-crystal $\mathrm{LiF}^{4,5}$, aluminum, and graphite ${ }^{6}$, and observed phase transitions with single-crystal boron nitride ${ }^{7}$. In these experiments, the intensity of the reflection was three orders of magnitude greater than the powder sample, allowing increased conjugate distances, and hence a film-protection capability. In the following decades, dynamic single-crystal shock experiments were performed using pulsed high- 
voltage sources ${ }^{8,9}$, notably by Rigg and Gupta ${ }^{10,11}$ who showed that the LiF strain mechanisms varied depending on whether the crystal lattice was shocked along the (100) or (111) crystal orientation.

Transformations of solid-state structures and compression dynamics that occur during shock experiments may be observed on very short, less than one ns, timescales by laser-driven plasma $\mathrm{x}$-ray sources ${ }^{12}$. Recently, the $\alpha-\varepsilon$ phase transition in single-crystal iron shocked along the (001) axis has been observed with a laser-driven plasma x-ray source $^{13,14}$. Also, Hawreliak et al. have reported nanosecond $\mathrm{x}$-ray diffraction from polycrystalline and amorphous materials using a laser-plasma $\mathrm{x}$-ray source with wavelengths in the range of 1.48 to $1.85 \AA^{15}$. The advantages of laser-driven plasma xray sources include short pulse width, reduction of bremsstrahlung emission, and a very small spot size compared to pulsed high-voltage electron beam sources. However, the capability of laser plasma sources to produce sufficient flux at penetrating $\mathrm{x}$-ray energies above $15 \mathrm{keV}$ may be cost-prohibitive ${ }^{16}$. In contrast, our low-cost, portable, single-pulse electron beam x-ray source will produce characteristic $17.4-\mathrm{keV}$ and $22.1-\mathrm{keV}$ line emission of sufficient intensity to perform a wide range of dynamic polycrystalline XRD experiments. Because these conventionally produced x-rays are sufficiently energetic, polyethylene blast shielding can be used to protect the detector and the x-ray source, and a vitreous carbon back window may be used to allow a shock-loaded steady-state observation time of greater than $100 \mathrm{~ns}$. A direct, comprehensive line flux emission comparison between high-voltage pulsed electron beam x-ray sources and laser-driven plasma x-ray sources has not been performed, and an experimental comparison would be very useful. 
This paper describes our newly designed, single-pulse XRD system. Because this system has a pinhole collimator and a small source spot size, it produces a highly collimated x-ray beam The 35-ns pulsed x-ray source provides sufficient characteristic line emission to observe polycrystalline Bragg diffraction patterns from flat, metal surfaces. High-energy-source bremsstrahlung shielding is incorporated directly into the xray diode, and Compton scattering of high-energy x-rays in the beam from the target sample and back window is the dominant contributor to background noise. A low-noise imaging system couples the phosphor image to a thermo-electrically cooled chargecoupled device (CCD) camera through a coherent fiber-optic bundle.

A series of high-explosives-driven shock-loaded polycrystalline experiments were performed on samples of tin and aluminum using this single-pulse XRD diagnostic. Analysis of the XRD images reflected from the shocked tin sample indicated that a phase transformation of the tin beta-phase into an amorphous or liquid state had occurred. Identical experiments with shock-loaded aluminum showed compression of the facecentered-cubic (fcc) lattice with no phase transformation.

\section{Experimental Apparatus}

National Security Technologies (NSTec) designed and built the 37-stage Marx bank having $8.1 \mathrm{nF}$ per stage described in this report. The capacitor stages were charged to $-30 \mathrm{kV}$ and subsequently triggered to discharge in series into a remote needle-andwasher $x$-ray diode through a $40-\Omega$, DS-2158 coaxial transmission line ${ }^{17}$. The Marx, coaxial transmission line, and x-ray diode were all pressurized to 80 psig with dry air. Upon triggering, the high stage-to-ground capacitance and low stage-to-stage capacitance 
cause sequential over-voltage of the ball gaps. This allows the Marx stages to erect in series, producing a high-voltage output. The stage ball gaps were independently adjusted to a spacing of $0.100 \pm 0.001 \mathrm{inch}$. The pulse width was approximately $35 \mathrm{~ns}$ with a pulse timing uncertainty less than $10 \mathrm{~ns}$. The $\mathrm{x}$-ray diode was located inside the blast containment vessel, with a specially designed high-voltage coaxial feed-through that allowed placement of the x-ray source very close to the sample. A vacuum feed-through penetration in the containment vessel was also required to evacuate the x-ray diode.

A schematic diagram of the single-pulse XRD experimental configuration appears in Figure 1. The x-ray diode was specially designed for $\mathrm{x}$-ray diffraction, with a short, low-characteristic-impedance vacuum transmission line section and a needle-and-washer anode-cathode (A-K) configuration ${ }^{9,18}$. A 1-cm-thick tungsten shield plate effectively eliminated direct high-energy bremsstrahlung emission outside the diode in all forward directions, except through the pinhole collimator. Because the molybdenum and silver emission line energies are much lower than the potential developed across the A-K gap, the diode was designed to reduce the load impedance. This resulted in higher emission current and lower electron impact energy, thereby increasing the production of characteristic line emission, while minimizing bremsstrahlung emission. A 5-mmdiameter stainless steel cathode and 1.5-mm-diameter anode of molybdenum or silver with spherical tips were used, and all sharp points and edges in the A-K region were eliminated. In this configuration, the peak voltage across the diode was estimated to be slightly less than $400 \mathrm{kV}^{19}$. Because the electron beam impact caused erosion of the anode surface, the anode was replaced after approximately five shots. The characteristic $\mathrm{K}_{\alpha}$ wavelengths and $\mathrm{K}_{\beta}$ filters for these anodes are shown in Table I. To produce a 
collimated x-ray beam, a tungsten pinhole was placed $70 \mathrm{~mm}$ from the anode. The smaller dimension of the rectangular pinhole was aligned parallel to the plane of dispersion to produce a collimated beam with a divergence of approximately $1^{\circ} \mathrm{FWHM}$. Because the diffraction line resolution is limited by the $1^{\circ}$ beam divergence in the scattering plane, the pinhole collimator was placed about $3 \mathrm{~cm}$ away from the axis of the target sample to maximize detector resolution.

$\mathrm{X}$-rays were detected by a thin, mirrored $\mathrm{P}-43$ phosphor that converted the $\mathrm{x}$-ray image to an optical image approximately $7.6 \mathrm{~cm}$ away from the sample. A half-inch tungsten beam-stop was used to prevent scattering from the direct beam onto the phosphor. Blast shielding was provided by two layers of 0.1 -in-thick polyethylene. The field of view was $18 \mathrm{~mm}$ wide by $12 \mathrm{~mm}$ high, allowing observations of $2 \theta_{\mathrm{B}}$ scattering angles in the range of $11^{\circ}$ to $25^{\circ}$. The optical image was transmitted to a thermoelectrically cooled CCD camera by a coherent fiber-optic bundle, and the images were recorded in a data file.

\section{Single-Pulse XRD Experiments}

Initial laboratory testing of the single-pulse XRD diagnostic system included a study of the material properties of the 1-mm-thick vitreous carbon used as a back window for dynamic shock loading. Vitreous carbon is an amorphous solid, and like a liquid, has no repeating structure. However, the minimum spacing of the atoms required by the Pauli exclusion principle results in a nonrandom probability distribution of the scattering centers. Therefore, coherent $\mathrm{x}$-ray scattering from local atomic configurations within amorphous solids or liquids causes broad interference peaks ${ }^{20}$. For example, Hawreliak et 
$a l .{ }^{13}$ have observed these broad interference peaks produced in Laue mode by coherent scattering from amorphous Metglas $\left[\mathrm{Ni}_{55} \mathrm{Fe}_{27} \mathrm{Co}_{15} \mathrm{Si}_{1.6} \mathrm{Al}_{1.4}\right]$ foil with a single pulse of 1.85- $\AA$ laser-plasma x-rays. A 0.561- $\AA$ single-pulse XRD image was obtained for a 1mm-thick sample of vitreous carbon with an $8.5^{\circ}$ input angle in a Bragg-reflection geometry using an image plate. The result is shown in Figure 2a, and a line-out of this image is shown in Figure 2b. The broad interference peaks are observed in first and second order, with the first interference peak observed at a scattering angle of approximately $15^{\circ}$.

Dynamic single-pulse XRD experiments were conducted at the high-explosives facility at NSTEc's Special Technologies Laboratory. The experimental package design for the two sample configurations described in this report are shown in Figure 3, and details of these experiments are summarized in Table II. A static single-pulse XRD image of the sample and a dark background image were obtained prior to the detonation. An electromagnetic pick-up at the x-ray diode end of the DS-2158 transmission determined the time at which the $\mathrm{x}$-ray pulse diffracted from the sample surface. Because vitreous carbon is opaque to visible light, the photon Doppler velocimetry diagnostic registered the time at which the shock reached the vitreous carbon-free surface. The shock velocity in vitreous carbon is approximately $4.92 \mathrm{~km} / \mathrm{s}^{9}$.

The first experiments were conducted with Detasheet high explosives on a 2-mmthick aluminum 6061-T6 buffer with a 1-mm vitreous carbon back window as shown in Figure 3a. The scattering angles for the three lowest-order fcc aluminum diffraction lines with $0.561-\AA \AA$ x-rays are given in Table III. As the shock front traverses the material interface, the shock wave partially reflects back into the aluminum, and partially 
transmits into the vitreous carbon back window. The penetration depth for $0.561-\AA \mathrm{x}-\mathrm{ray}$ in the aluminum sample was approximately $0.4 \mathrm{~mm}$, and the reflected shock must exceed the x-ray penetration depth to ensure equal pressure for all aluminum x-ray reflections. Also, the x-ray pulse must fire before the transmitted shock in the vitreous carbon reflects from the free surface back to the aluminum-vitreous carbon interface. At the time of the $\mathrm{x}$-ray interrogation, the pressure at the interface is substantially reduced compared to the peak pressure that occurs when the shock first arrives at the interface. The peak pressure and the reduced pressure at the X-ray arrival time were calculated by the WONDY code, and are given for experimental run $\# 070607$ in Table II. From this code, we estimated that the reflected shock had reached a depth of $0.7 \mathrm{~mm}$ in the shocked aluminum, measured from the aluminum-vitreous carbon interface, exceeding the x-ray penetration depth. The interface had moved approximately $0.165 \mathrm{~mm}$ in the direction of the initial shock. The shift of the (200) and (220) reflections in the image plane caused by the shocked aluminum compression and the translation of the aluminum-vitreous carbon interface are shown in Figure 4. We also observed that the x-ray reflections from the vitreous carbon back window are essentially unchanged in the dynamic image. After correcting for the translation, we measured the shock-loaded aluminum compression to be $\Delta l / l=-0.020 \pm 0.006$.

Experiments to study the dynamic properties of tin were conducted with a thin 0.106-mm sheet of tin sandwiched between the 6061-T6 aluminum buffer and the 1-mm vitreous carbon back window as shown in Figure 3b. Experiment \#070605-1 used a silver anode and palladium filter to produce $0.561-\AA$ x-rays. At this wavelength, $90 \%$ of the diffraction occurs within $0.007 \mathrm{~mm}$ of the tin-vitreous carbon interface. The room- 
temperature tin $\beta$-phase structure is face-centered-tetragonal with a lattice constant of $3.21 \AA$ and a c/a ratio of 0.546 . A second identical lattice is offset at coordinates $(a, a, c) / 4$. The four lowest-order diffraction lines are given in Table IV. The (311) and (400) diffraction lines are not resolved by our diffraction system, but are quite intense, and clearly indicate the presence of the tin $\beta$-phase structure. The experimental timing, peak pressure, and reduced pressure as calculated by the WONDY code for experiment \#070605-1 are shown in Table II. X-ray diffraction was observed approximately $80 \mathrm{~ns}$ after the initial shock arrived at the tin-vitreous carbon interface.

Figure 5a shows the dynamic-minus-static $0.561-\AA$ XRD image of the tin sample, and the line-out is shown in Figure 5b. By subtracting the static image from the dynamic image, the fixed pattern noise within the CCD camera is reduced, and the vitreous carbon back window interference peak is eliminated, because changes in the reflections from the vitreous carbon caused by dynamic effects are insignificant. Therefore, the resulting dynamic-minus-static image enhances the signal-to-noise ratio of the tin x-ray reflections. The strong, unresolved (400) and (311) lines disappear, and are replaced with a broad peak at the low-scattering-angle edge of the field of view. The broad peak in our image is compared to a static liquid tin XRD experiment performed by Vahvaselkä[21] in Figure $5 b$. The agreement of the width and location of the broad interference peak leads to the conclusion that the shock-loaded tin has transformed to an amorphous or liquid state. The slight discrepancies in the location of the broad interference peak and Vahvaselkä's results can be explained by our system resolution, the small translation of the tin-vitreous carbon interface, and possibly the increased density caused by compression of the amorphous or liquid tin. 
Experiment $\# 070605-1$, performed on tin samples with 0.561- $\AA$ x-rays was repeated with a molybdenum anode and zirconium filter at a wavelength of $0.709 \AA$ in experiment \#070605-2, and details are given in Table II. At this wavelength the $\mathrm{x}$-ray penetration depth in tin is about $0.015 \mathrm{~mm}$. No other changes were made to the experimental package, timing, or diagnostics. The x-ray pulse arrived at the tin-vitreous carbon interface approximately $100 \mathrm{~ns}$ after the incident shock, and the dynamic-minusstatic image is shown in Figure 6. Compared with those in Figure 5, the unresolved static (311) and (400) diffraction lines in Figure 6 are observed at larger scattering angles due to the longer wavelength x-rays. Again, we observe a broad interference peak in the dynamic-minus-static image near the center of the detector's field of view, with the location and width of the peak in good agreement with the static liquid tin diffraction experiments of Vahvaselkä ${ }^{21}$ as shown in Figure $6 b$.

\section{Conclusions}

We have shown that shock-loaded tin driven to peak pressures above $175 \mathrm{kbar}$ transforms to a liquid or amorphous state upon release to a reduced pressure around 55 kbar. Mabire and Héreil ${ }^{22}$ have performed shock-loaded tin experiments with a single crystal $\mathrm{LiF}$ as a back window using a projectile launcher. For these experiments, velocity interferometer for any reflector (VISAR) diagnostics were used to determine the tin-LiF interface velocity, and polymorphic transitions and melt-on-release were inferred from the velocity profiles. In shot number SN02, Mabire and Héreil report a peak impact stress of 17.9 GPa at the interface, which causes a tin phase transformation into the bodycentered-tetragonal or $\gamma$-phase at about 100 kbar. Upon release, the sample returns to the 
$\beta$-phase, as shown in their temperature-stress phase diagram, with temperatures at least $200 \mathrm{~K}$ below transformation to the liquid phase. Their shock-loaded tin experiment, shot number SN02, was driven to a pressure very close to the peak pressure calculated by WONDY code for our tin experiments. Therefore, our observation of a shocked-tin phase transition to a liquid or amorphous state is not supported by the VISAR experiments of Mabire and Héreil, and the reason for the ambiguity is not clear.

We expect to continue similar experiments with an improved single-pulse XRD system in the near future. Improvements will include a large-format image plate detection system for observation of higher-order diffraction lines. A beam block will permit partial transmission of the direct beam onto the image plate, improving the data analysis capability. Improvements will also be made to the x-ray shielding and collimation. The single-pulse XRD is expected to become a valuable tool for determination of dynamic phase transitions. 
${ }^{1}$ W. J. Murphy, A. Higginbotham, J. S. Wark, N. Park, Shock Compression of Condensed Matter - 2007, edited by M. Elert, M. D. Furnish, R. Chau, N. Holmes, and J. Nguyen, (American Institute of Physics, 2007), p. 325.

${ }^{2}$ D. V. Morgan, D. R. Macy, M. J. Madlener, and J. G. Morgan, 2007 IEEE Pulsed Power Conference, 209 (2007).

${ }^{3}$ Q. Johnson, R. N. Keeler, and J. W. Lyle, Nature 213, 1114 (1967).

${ }^{4}$ Q. Johnson, A. Mitchell, R. Keeler, and L. Evans, Phys. Rev. Lett. 25, 1099 (1970).

${ }^{5}$ Q. Johnson, A. Mitchell, and L. Evans, Nature 231, 311 (1971).

${ }^{6}$ Q. Johnson and A. C. Mitchell, and L. Evans, Appl. Phys. Lett. 21, 29 (1972).

${ }^{7}$ Q. Johnson, and A. C. Mitchell, Phys. Rev. Lett. 29, 1369 (1972).

${ }^{8}$ T. d'Almeida and Y. M. Gupta, Phys. Rev. Lett. 85, 330 (2000).

${ }^{9}$ Y. M. Gupta, K. A. Zimmerman, P. A. Rigg, E. B. Zaretsky, D. M. Savage, and P. M. Bellamy, Rev. Sci. Instrum. 70, 4008 (1999).

${ }^{10}$ P. A. Rigg and Y. M. Gupta, Appl. Phys. Lett. 73, 1655 (1998).

${ }^{11}$ P. A. Rigg and Y. M. Gupta, Phys. Rev. B 63, 094112 (2001).

${ }^{12}$ J. S. Wark, R. R. Whitlock, A. A. Hauer, J. E. Swain, and P. J. Solone, Phys Rev. B 40, 5705 (1989).

${ }^{13}$ J. Hawreliak, J. D. Colvin, J. H. Eggert, D. H. Kalantar, H. E. Lorenzana, J. S. Stölken, H. M. Davies, T. C. Germann, B. L. Holian, K. Kadau, P. S. Lomdahl, A.

Higginbotham, K. Rosolankova, J. Sheppard, and J. S. Wark, Phys. Rev. B 74, 184107 (2006). 
${ }^{14}$ D. H. Kalantar, G. W. Collins, J. D. Colvin, J. H. Eggert, J. Hawreliak, H. E. Lorenzana, M. A. Meyers, R. W. Minich, K. Rsolankova, M. S. Schneider, J. S. Stölken, and J. S. Wark, International Journal of Impact Engineering 33, 343 (2006).

${ }^{15}$ J. Hawreliak, H. E. Lorenzana, B. A. Remington, S. Lukezic, J. S. Wark, Rev. Sci. Instrum. 78, 083908 (2007).

${ }^{16}$ H.-S. Park, D. M. Chambers, H.-K. Chung, R. J. Clarke, R. Eagleton, E. Giraldez, T. Goldsack, R. Heathcote, N. Izumi, M. H. Key, J. A. King, J. A. Koch, O. L. Landen, A. Nikroo, P. K. Patel, D. F. Price, B. A. Remington, H. F. Robey, R. A. Snavely, D. A. Steinman, R. B. Stephens, C. Stoeckl, M. Storm, M. Tabak, W. Theobald, R. P. J. Town, J. E. Wickersham, and B. B. Zhang, Phys. Plasmas 13, 056309 (2006).

${ }^{17}$ D. Platts, M. Hockaday, D. Beck, W. Coulter, R. C. Smith, Proceedings of the $10^{\text {th }}$ IEEE International Pulsed Power Conference 892 (1995).

${ }^{18}$ Q. Johnson, A. C. Mitchell, and I. D. Smith, Rev. Sci. Instrum. 51, 741 (1980).

${ }^{19}$ R. J. Commisso, J. R. Boller, G. Cooperstein, M. C. Myers, S. B. Swanekamp, and F. C. Young, Naval Research Laboratory Pulsed Power Physics Technote No. 2000-16, 2000 (unpublished).

${ }^{20}$ B. E. Warren, X-ray Diffraction, (Dover Publication, Inc., New York, 1990), p. 120.

${ }^{21}$ K. S. Vahvaselkä, Physica Scripta 18, 266 (1978).

${ }^{22}$ C. Mabire and P. L. Héreil in Shock Compression of Condensed Matter - 1999, edited by M. D. Furnish, L. C. Chhabildas, and R. S. Hixson (American Institute of Physics, 1999), p. 93. 


\section{ACKNOWLEDGMENTS}

This manuscript has been authored by National Security Technologies, LLC, under Contract No. DE-AC52-06NA25946 with the U.S. Department of Energy. The United States Government retains and the publisher, by accepting the article for publication, acknowledges that the United States Government retains a non-exclusive, paid-up, irrevocable, world-wide license to publish or reproduce the published form of this manuscript, or allow others to do so, for United States Government purposes. 
TABLE I. Characteristic emission wavelengths for silver and molybdenum anodes.

\begin{tabular}{ccc}
\hline \hline Anode & $\mathrm{K}_{\alpha}$ Wavelength $(\AA)$ & $\mathrm{K}_{\beta}$ Line Emission Filter \\
\hline $\operatorname{Mo}(\mathrm{Z}=42)$ & 0.709 & $0.050 \mathrm{~mm} \mathrm{Zr}$ \\
$\operatorname{Ag}(\mathrm{Z}=47)$ & 0.561 & $0.025 \mathrm{~mm} \mathrm{Pd}$ \\
\hline \hline
\end{tabular}


TABLE II. Summary of Dynamic Experiments.

\begin{tabular}{|c|c|c|c|c|}
\hline Experiment \# & Configuration & $\begin{array}{c}\text { X-ray delay from } \\
\text { interface shock arrival } \\
\text { (ns) }\end{array}$ & $\begin{array}{l}\text { Peak interface pressure } \\
\text { (kbar) }\end{array}$ & Release pressure (kbar) \\
\hline 070607 & $\mathrm{Al}: \mathrm{C}$ & 90 & - & 64 \\
\hline $070605-1$ & $\mathrm{Al}: \mathrm{Sn}: \mathrm{C}$ & 190 & 175 & 58 \\
\hline $070605-2$ & $\mathrm{Al}: \mathrm{Sn}: \mathrm{C}$ & 100 & 193 & 64 \\
\hline
\end{tabular}


TABLE III. X-ray reflections from fcc aluminum at room temperature and pressure.

\begin{tabular}{ccc}
\hline \hline Miller Indices & d-spacing $(\AA)$ & $2 \theta_{\mathrm{B}}\left(^{\circ}\right), \lambda=0.561 \AA$ \\
\hline$(111)$ & 2.35 & 13.7 \\
$(200)$ & 2.04 & 15.8 \\
$(220)$ & 1.44 & 22.4 \\
\hline \hline
\end{tabular}


TABLE IV. X-ray reflections from $\beta$-phase tin at room temperature and pressure.

\begin{tabular}{cccc}
\hline \hline Miller Indices & d-spacing $(\AA)$ & $2 \theta_{\mathrm{B}}\left(^{\circ}\right), \lambda=0.709 \AA$ & $2 \theta_{\mathrm{B}}\left({ }^{\circ}\right), \lambda=0.561 \AA$ \\
\hline$(220)$ & 2.91 & 14.0 & 11.1 \\
$(111)$ & 2.79 & 14.6 & 11.6 \\
$(400)$ & 2.06 & 19.8 & 15.7 \\
$(311)$ & 2.01 & 20.3 & 16.0 \\
\hline \hline
\end{tabular}




\section{FIGURE CAPTIONS}

FIG. 1. Schematic diagram of the single-pulse XRD system. Filtered x-rays from the anode are collimated by the tungsten pinhole with an angular divergence of $1^{\circ}$. The diffracted x-rays are detected by a phosphor screen $7.6 \mathrm{~cm}$ from the sample axis.

FIG. 2. (a) XRD pattern from a 1-mm-thick sheet vitreous or "glassy" carbon with 0.561$\AA$ x-rays at an input angle of $8.5^{\circ}$ with respect to the surface. An image plate is used for X-ray detection of the first and second interference peaks. (b) Interference peak integrated intensity as a function of scattering angle.

FIG. 3. Experimental designs for (a) shock-loaded aluminum and (b) shock-loaded tin.

FIG. 4. Static and dynamic aluminum (111), (200), and (220) XRD reflections observed with $0.561-\AA$ x-rays. The shift to larger scattering angles is caused primarily by lattice compression. The first interference peak from the vitreous carbon is observed at a scattering angle of approximately $15^{\circ}$.

FIG. 5. (a) Dynamic-minus-static image for shock-loaded tin diffraction at $0.561 \AA$. This image-processing technique allows noise reduction by cancellation of fixed-pattern noise within the CCD. The coherent scattering from the amorphous vitreous carbon is also cancelled, because dynamic shock effects on this material are shown to be small. (b) 
line-out of the dynamic-minus-static image; the solid line, liquid tin diffraction results, is from Vahvaselkä[21].

FIG. 6. (a) Dynamic-minus-static image for shock-loaded tin at $0.709 \AA$. The entire firstorder broad interference peak from amorphous tin is in the field of view. (b) $\boldsymbol{\Lambda}$, line-out of dynamic-minus-static integrated intensity; the solid line, liquid tin diffraction results, is from Vahvaselkä[21]. 


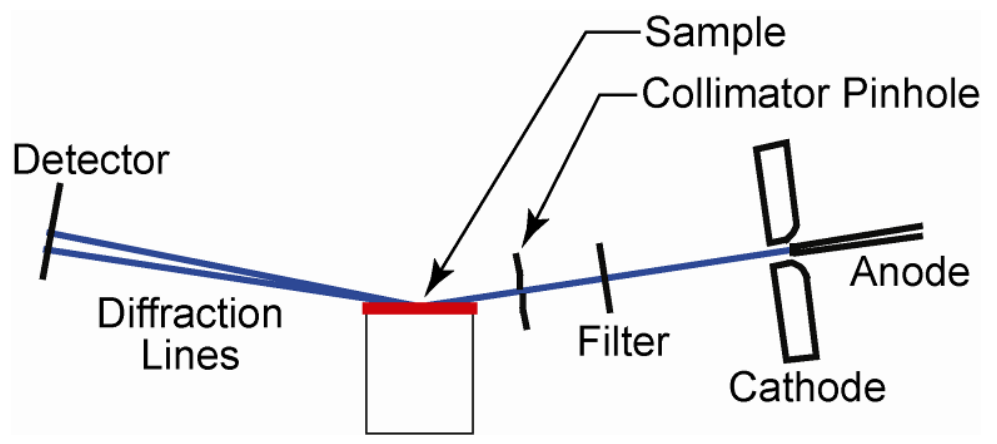

Figure 1

(a) $\begin{gathered}\text { First } \\ \text { Interference Peak }\end{gathered}$

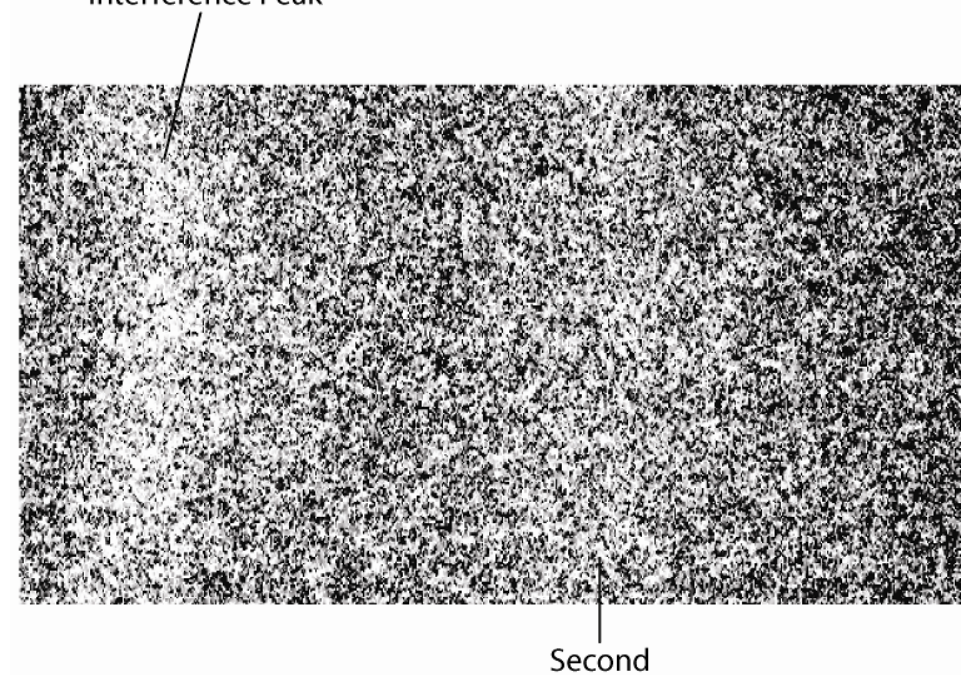

Interference Peak

(b)

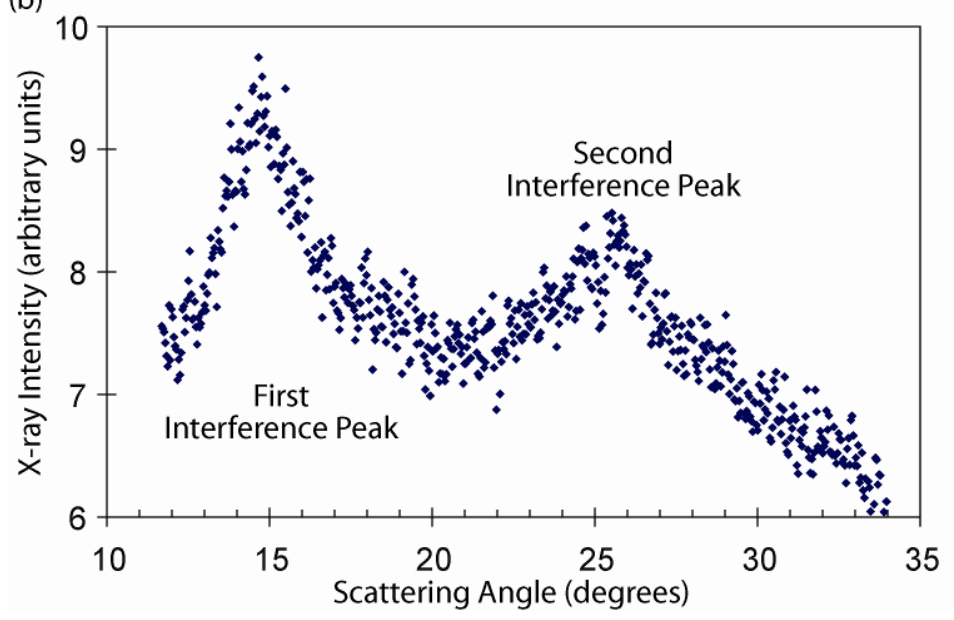

Figure 2 
(a) Diffracted

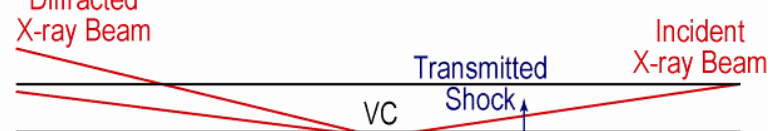
Maximum X-ray Penetration - - - - - - $\uparrow \mid$ Reflected Shock
Depth in Aluminum

Al Incident Shock

(b)

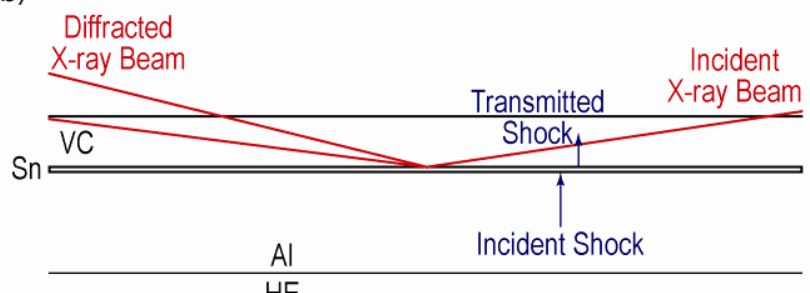

Figure 3

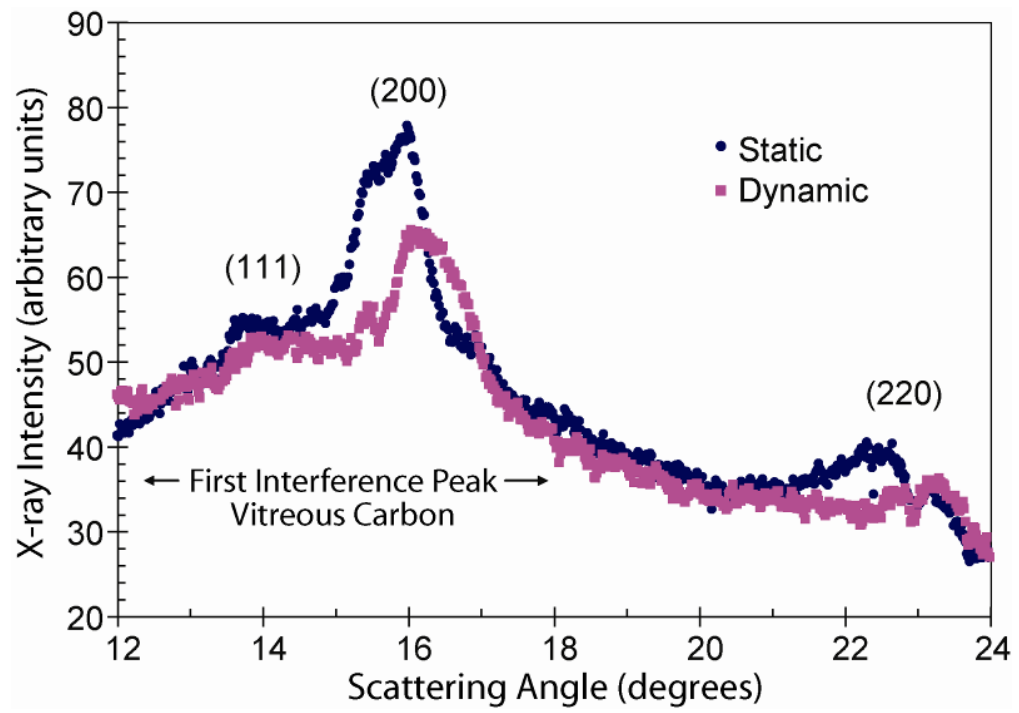

Figure 4 
(a)

(311)

(400)

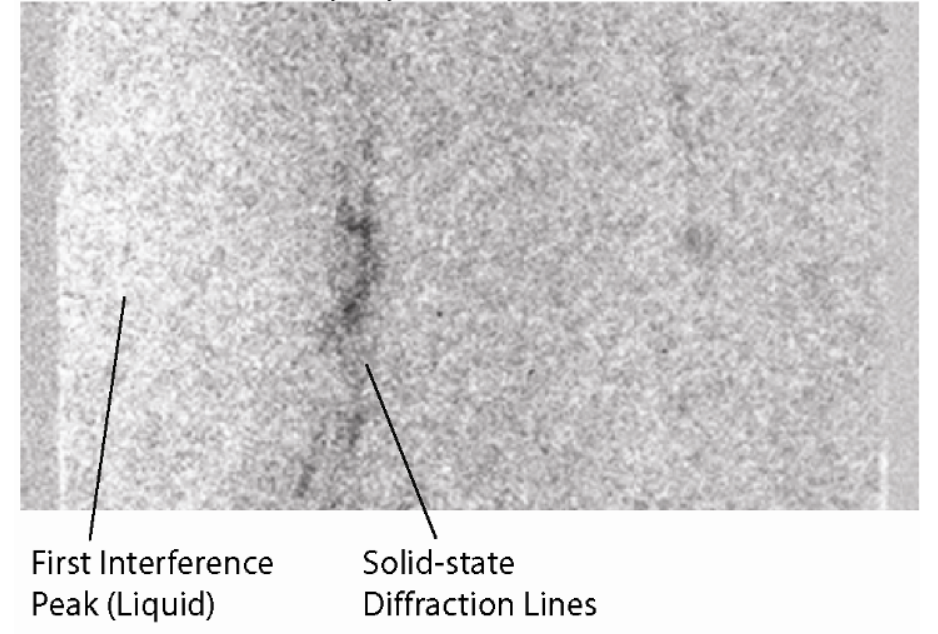

(b)

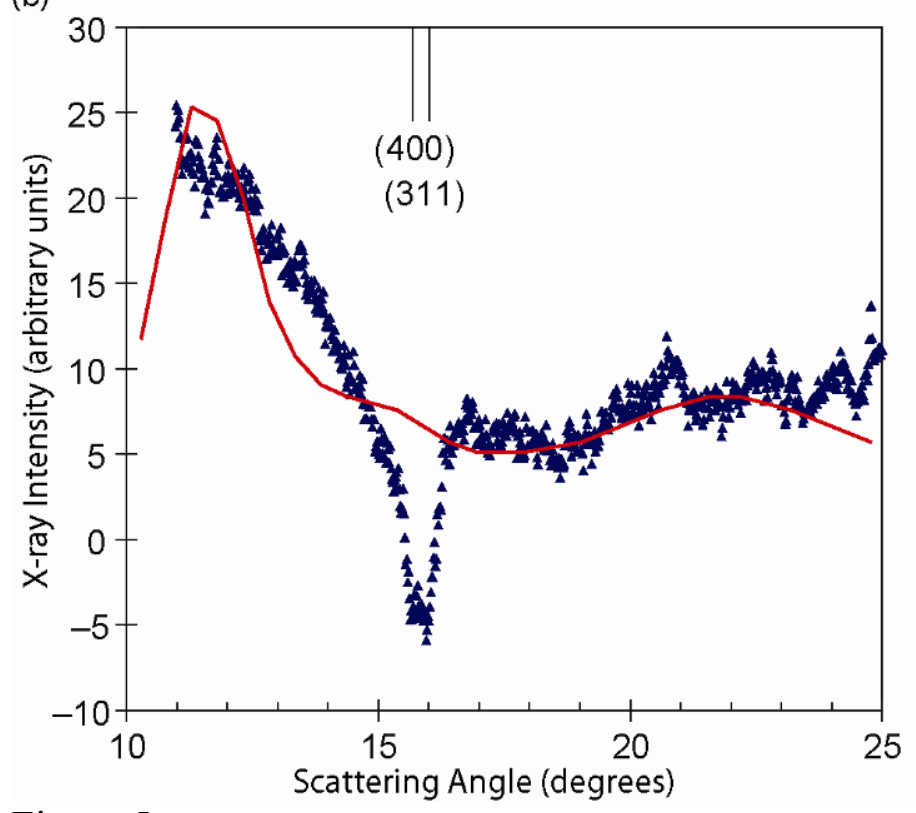

Figure 5 
(a)

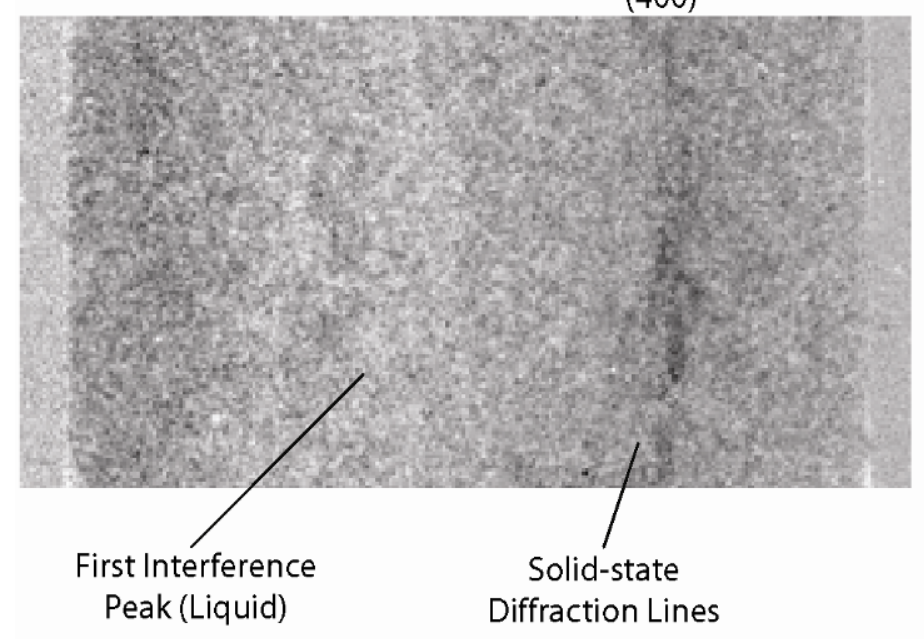

(b)

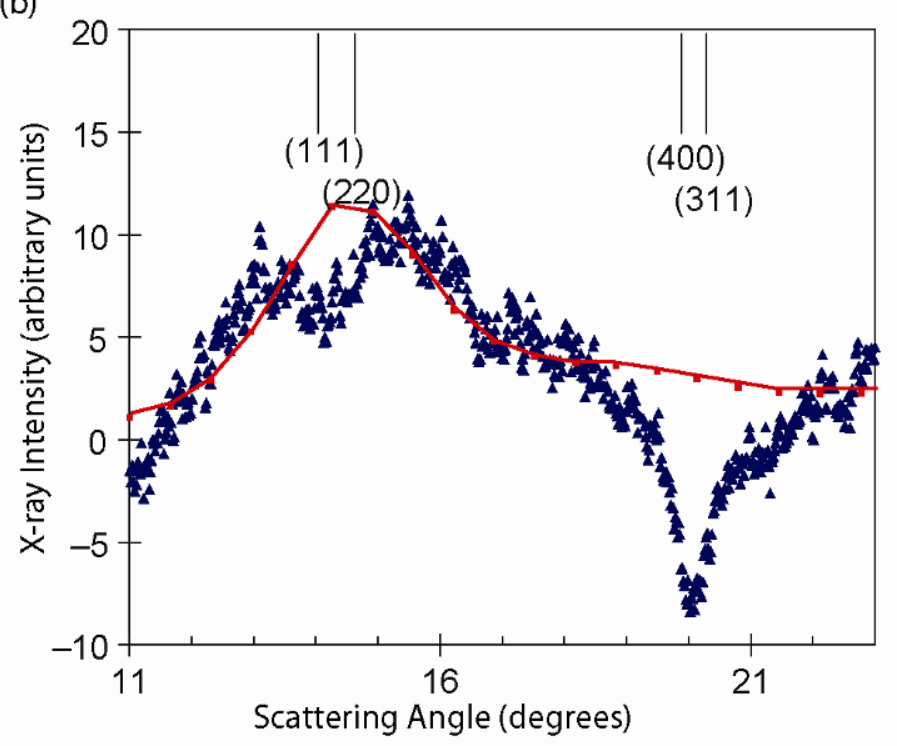

(311)

(400)

Solid-state

Diffraction Lines

Figure 6 